\title{
Images of the month 3: Bilateral group B Streptococcus endophthalmitis - more than meets the eye
}

\author{
Authors: Ray Peng Khoon Peh, ${ }^{\mathrm{A}}$ Hui Zhuan Tan, ${ }^{\mathrm{B}}$ Zhong Hong Liew, ${ }^{\mathrm{C}}$ Jin Rong Low, ${ }^{\mathrm{D}}$ Marjorie Foo ${ }^{\mathrm{E}}$ and Cynthia Ciwei Lim ${ }^{\mathrm{F}}$
}

Fig 1. Photograph of right eye showed conjunctival injection with fine keratic precipitates and hypopyon in the anterior chamber. The left eye was similarly affected, but with a greater degree of conjunctival injection and oedema.

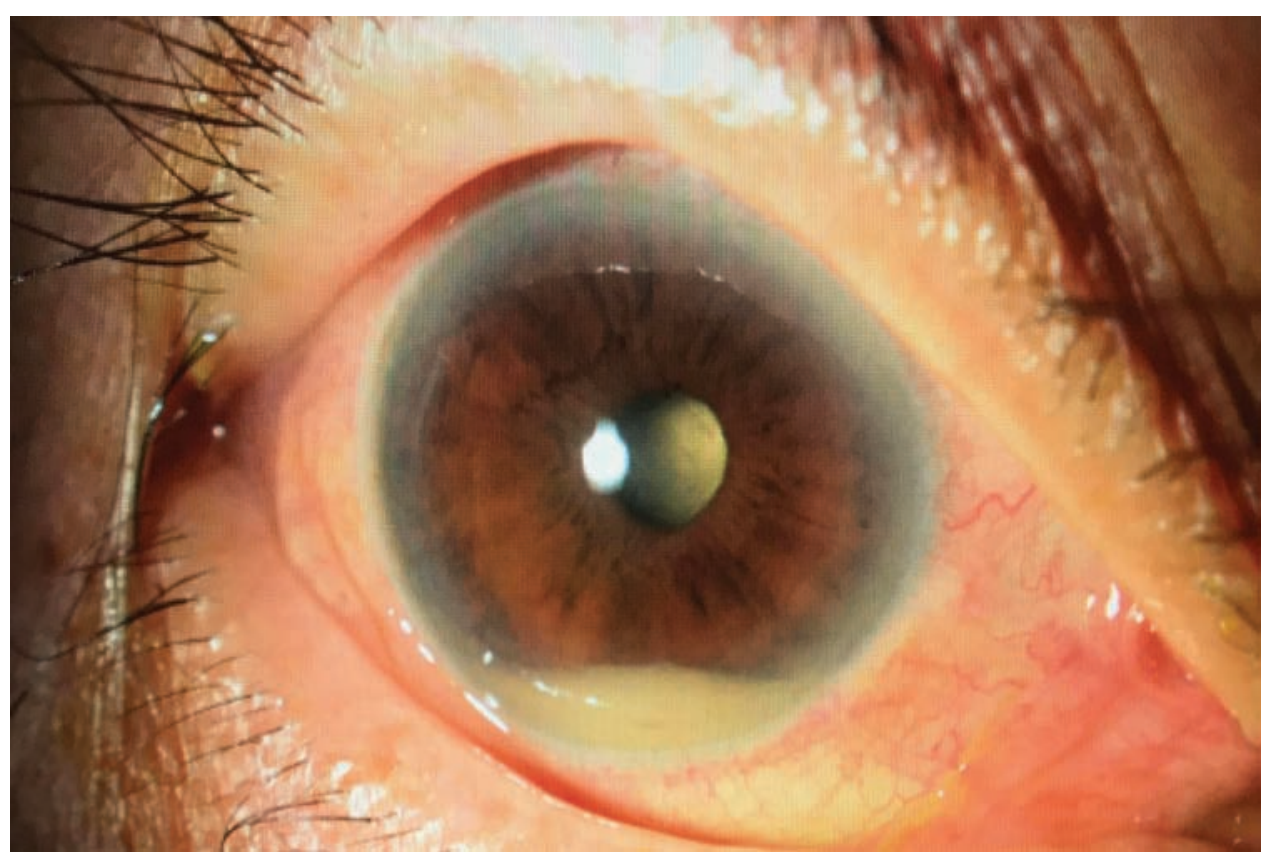

KEYWORDS: Eye, chronic renal failure, bacteraemia, abscess, central line

\section{Case presentation}

A 60-year-old diabetic on haemodialysis for end-stage renal failure presented with pain and discharge in both eyes over 2 days,

Authors: A resident, Department of Renal Medicine, Singapore General Hospital, Singapore; ${ }^{B}$ senior resident, Department of Renal Medicine, Singapore General Hospital, Singapore; ' senior resident, Department of Renal Medicine, Singapore General Hospital,

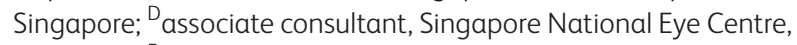
Singapore; 'E senior consultant, Department of Renal Medicine, Singapore General Hospital, Singapore; ${ }^{F}$ consultant, Department of Renal Medicine, Singapore General Hospital, Singapore associated with fever and chills. She had severe loss of visual acuity in both eyes and could only perceive light. Slit lamp examination revealed bilateral keratic precipitates in the cornea and fibrin and hypopyon in the anterior chamber (Fig 1). Cultures from intravitreal pus from both eyes were positive for group B Streptococcus (GBS). Physical examination was remarkable for an erythematous $3 \times 3 \mathrm{~cm}$ fluctuant swelling of the right chest wall at the exit site of her tunnelled dialysis catheter. Surgical incision and drainage of the tunnel tract abscess with removal of the catheter was performed. Abscess fluid and catheter tip cultures were positive for GBS, while blood cultures were positive for GBS and Enterococcus faecalis. Echocardiography and thoracic computed tomography found an infected thrombus in the right internal jugular vein and superior vena cava. There were no vegetations on the cardiac valves. Her vision did not improve despite emergent treatment with empirical then culture-directed intravitreal and systemic antibiotics.

Endogenous bacterial endophthalmitis is caused by transmission of bacteria from a distant organ to the eye by haematogenous spread and is associated with diabetes mellitus, intravenous drug use and immunocompromised states. ${ }^{1}$ GBS, a common 
commensal in the skin, perineum and upper respiratory tract, is a rare cause of endogenous endophthalmitis, ${ }^{2}$ with only 20 cases $(6 \%)$ among 342 cases of endogenous bacterial endophthalmitis reported between 1986 and 2012. ${ }^{1}$ Afflicted individuals frequently had rapid, severe and irreversible vision loss. ${ }^{3}$

This unusual case of bilateral endogenous GBS endophthalmitis as the first presentation of a dialysis catheter tunnel tract abscess highlighted the need to recognise possible infective complications of dialysis catheter-related bloodstream infections in diabetic end-stage kidney failure. The devastating permanent vision loss also underscored the need to avoid catheters for dialysis vascular access where possible. ${ }^{4}$.

\section{References}

1 Jackson TL, Paraskevopoulos T, Georgalas I. Systematic review of 342 cases of endogenous bacterial endophthalmitis. Surv Ophthalmol 2014;59:627-35.
2 Wong J-S, Chan T-K, Lee H-M, Chee S-P. Endogenous bacterial endophthalmitis: an east Asian experience and a reappraisal of a severe ocular affliction. Ophthalmology 2000;107:1483-91.

3 Lee S-Y, Chee S-P. Group B Streptococcus endogenous endophthalmitis: case reports and review of the literature. Ophthalmology 2002:109:1879-86.

4 Vascular Access 2006 Work Group. Clinical practice guidelines for vascular access. Am J Kidney Dis 2006;48(Suppl 1):S176-247.

Address for correspondence: Cynthia C Lim, Department of Renal Medicine, Singapore General Hospital, Academia Level 3, 20 College Road, Singapore 169856.

Email: cynthia.lim.c.w@singhealth.com.sg

\section{Outpatients: the future - adding value through sustainability}

Published in 2018, this report from the Royal College of Physicians argues for

new models of outpatient care.

Download the report and

supplementary case studies at

www.rcplondon.ac.uk/outpatients-future-sustainability 